\title{
Letter
}

\section{Beltrami fields in a hot electron-positron-ion plasma}

\author{
M. IQBAL ${ }^{1}$ and P. K. SHUKLA ${ }^{2}$ \\ ${ }^{1}$ Department of Physics, University of Engineering and Technology, Lahore 54890, Pakistan \\ (iqbal_uot@yahoo.com) \\ ${ }^{2}$ RUB International Chair, International Centre for Advanced Studies in Physical Sciences, Institut für Theoretische Physik, \\ Fakultät für Physik und Astronomie, Ruhr-Universität Bochum, D-44780 Bochum, Germany
}

(Received 13 September 2011; revised 27 December 2011; accepted 3 January 2012; first published online 6 February 2012)

\begin{abstract}
A possibility of relaxation of relativistically hot electron and positron $(e-p)$ plasma with a small fraction of hot or cold ions has been investigated analytically. It is observed that a strong interaction of plasma flow and field leads to a non-force-free relaxed magnetic field configuration governed by the triple curl Beltrami (TCB) equation. The triple curl Beltrami (TCB) field composed of three different Beltrami fields gives rise to three multiscale relaxed structures. The results may have the strong relevance to some astrophysical and laboratory plasmas.
\end{abstract}

A single fluid magnetized plasma, under certain topological constraints, have the potential to self-organize to a state of minimum energy. This minimum energy state, also called as Taylor state (Taylor 1974, 1986), is characterized by Beltrami eigenfunctions $\nabla \times \boldsymbol{B}=\lambda \boldsymbol{B}$ (Yoshida and Giga 1990). The multiple Beltrami fields may be created when a higher order effect (singular perturbation) is introduced (Yoshida and Mahajan 1999). For instance, a collisionless Hall effect produces double Beltrami field (Mahajan and Yoshida 1998; Yoshida et al. 2001; Shukla and Mahajan 2004a, b; Shukla 2005). The inertial effects can also introduce couplings of Beltrami fields (Bhattacharyya et al. 2003; Iqbal and Shukla 2011). The relaxed equilibrium of multifluid plasmas exhibits strong flow and pressure gradients, which are essential features of all practical plasmas. On the other hand, relaxed state governed by single Beltrami field is a stationary force-free state, and cannot be used to study the high $\beta$ (a ratio of kinetic to magnetic pressures) diamagnetic relaxed states of plasmas.

In this letter, we study the Beltrami fields to be created in relativistically hot electron, positron and ion $(e-p-i)$ plasmas. The relativistic electron-positron $(e-p)$ plasmas are important as such plasmas are found, for example, in pulsars (Chen and Ruderman 1993; Machabeli et al. 1999, 2000a, b, 2002), in active galactic nuclei (Lightman and Zdziarski 1987; Svensson 1994), in black hole accretion disks (Takahara and Kusonose 1985; Björsson and Svensson 1992), in the large scale jets associated with the galaxy M87 (Reynolds et al. 1996) and so on. The relativistic $e-p$ plasmas are also believed to exist at the early stage of universe (Misner et al. 1980; Rees 1983). Furthermore, it is possible to produce relativistic $e-p$ pairs in laboratory with the help of ultraintense laser pulses (Liang et al. 1998; Marklund and Shukla 2006). The production of electron-positron pairs in large tokamaks have been discussed by Helander and Ward (2003) where the collisions between high energy electrons and thermal particles may create abundant amount of positrons. The possibility of creating electron-positron pairs by intense laser pulses impinged onto a slightly overdense electronion plasmas have been investigated by Berezhiani et al. (2007).

A small fraction of ions, which is thought to be likely to present in addition to $e-p$ pair in astrophysical and cosmic plasmas, introduces new and interesting properties to the composite system. The three component $(e-p-i)$ plasmas are important regarding pulsar magnetosphere and have been studied extensively (Lakhina and Buti 1981; Lominadze et al. 1986; Rizzato 1989; Berezhiani et al. 1994). Such plasmas can be created by the propagation of intense short laser pulses in a plasma (Berezhiani et al. 1992a). The three component plasmas have also been found in experiments where positrons were used to study transport in tokamaks (Surko et al. 1986; Surko and Murphy 1990; Tinkle et al. 1994). The $e-p-i$ plasmas, due to their importance and applications, have been a topic of considerable interest. Berezhiani et al. (1992b) studied the effect of positrons on the wakefields generated in three species plasma consisting of electrons, positrons and ions. The creation of stable localized structures of relativistically strong EM radiation in hot $e-p-i$ plasma have been investigated by Berezhiani and Mahajan (1994, 1995). The positrons are found to reduce the amplitudes of ionacoustics solitons in electron-positron and ion plasmas by Popel et al. (1995). Shatashvili et al. (1997) have studied the nonlinear wave dynamics in two temperature electron-positron-ion plasma. Solitons and ion acoustics shock waves in unmagnetized hot $e-p-i$ plasmas are investigated by Mahmood and Rehman (2009) and Shah 
and Saeed (2009), respectively. Mostly the collective and nonlinear phenomena in relativistic $e-p-i$ plasmas have been studied whereas the self-organization of such plasmas have not got much attention. It has been shown that relativistically hot $e-p$ plasma can relax to Beltrami fields (Iqbal et al. 2008) but what about relativistically hot $e-p-i$ plasmas? Is it possible for a hot $e-p$ plasma having a small fraction of heavy ions to self-organize to Beltrami fields. In this letter, we have addressed this problem and it is shown that hot $e-p$ plasmas with small number of ions can also relax to Beltrami fields.

By hot $e-p-i$ plasmas, we mean that the thermal energy of the plasma particles is of the same order or larger than the rest mass energy. If the velocity distribution of the particles is taken to be a local relativistic Maxwellian, the relative temperature effect can be expressed by the factor $G_{\alpha}\left(z_{\alpha}\right)=K_{3}\left(1 / z_{\alpha}\right) / K_{2}\left(1 / z_{\alpha}\right)$, where $K$ 's are modified Bessel functions, and $z_{\alpha}=T_{\alpha} / m_{0 \alpha} c^{2} ; m_{0 \alpha}$ and $T_{\alpha}$ are the particle invariant rest mass and temperature, respectively. For nonrelativistic temperatures $T_{\alpha} \ll m_{0 \alpha} c^{2}$, and $G_{\alpha}=1+5 T_{\alpha} / 2 m_{0 \alpha} c^{2}$; and for highly relativistic temperatures $T_{\alpha} \gg m_{0 \alpha} c^{2}$, and $G_{\alpha}=4 T_{\alpha} / m_{0 \alpha} c^{2}$. The hydrodynamics of the fluids of species $\alpha(\alpha=e, p, i)$ is given as (Berezhiani et al. 2002):

$$
\frac{\partial}{\partial t}\left(G_{\alpha} m_{0 \alpha} \gamma_{\alpha} \boldsymbol{V}_{\alpha}\right)+m_{0 \alpha} c^{2} \nabla\left(G_{\alpha} \gamma_{\alpha}\right)=e_{\alpha} \boldsymbol{E}+\boldsymbol{V}_{\alpha} \times \boldsymbol{\Omega}_{\alpha},
$$

and

$$
\frac{\partial}{\partial t} \boldsymbol{\Omega}_{\alpha}=\nabla \times\left(\boldsymbol{V}_{\alpha} \times \boldsymbol{\Omega}_{\alpha}\right),
$$

where the generalized vorticity $\boldsymbol{\Omega}_{\alpha}$ is given by

$$
\boldsymbol{\Omega}_{\alpha}=\nabla \times\left(G_{\alpha} m_{0 \alpha} \gamma_{\alpha} \boldsymbol{V}_{\alpha}\right)+\frac{e_{\alpha}}{c} \boldsymbol{B} .
$$

The fluid velocity is represented by $\boldsymbol{V}_{\alpha}$, electric and magnetic fields are represented by $\boldsymbol{E}$ and $\boldsymbol{B}$, respectively. $e_{\alpha}$ is the charge of $\alpha$ - species, $\gamma_{\alpha}=\left(1-\boldsymbol{V}_{\alpha}^{2} / c^{2}\right)^{-1 / 2}$ is the relativistic factor, and $c$ is the velocity of light. The thermal pressure $P_{\alpha}\left(=n_{\alpha} T_{\alpha} / \gamma_{\alpha}\right)$ appears in (1) through the temperature dependent factor $G_{\alpha}$ defined by $\gamma_{\alpha} \nabla P_{\alpha}=m_{0 \alpha} c^{2} n_{\alpha} \nabla G_{\alpha}$, where $n_{\alpha}$ is the density in the laboratory frame of the fluid of species $\alpha$.

We consider the quasineutral plasma where the density is taken to be constant for simplicity and velocities of fluid elements are nonrelativistic $\left[\gamma_{\alpha} \simeq 1+V_{\alpha}^{2} / 2 c^{2}\right]$. We also assume that temperatures of hot electrons and positrons are equal and constant that is $T_{e}=T_{p}=T_{1}$ $\left(G_{e}=G_{p}=G_{1}\right)$. The temperature of ions is taken to be different from that of electrons and positrons and is equal to $T_{i}=T_{2}\left(G_{i}=G_{2}\right)$.

The simplest steady state solution to (2) is given by the 'Beltrami conditions' which require the alignment of generalized vorticities and corresponding flows, that is

$$
\boldsymbol{\Omega}_{\alpha} \| \boldsymbol{V}_{\alpha} \quad(\alpha=e, p, i)
$$

where

$$
\begin{aligned}
& \boldsymbol{\Omega}_{e}=\nabla \times\left(G_{1} m_{0 e} \boldsymbol{V}_{e}\right)-\frac{e}{c} \boldsymbol{B}, \\
& \boldsymbol{\Omega}_{p}=\nabla \times\left(G_{1} m_{0 p} \boldsymbol{V}_{p}\right)+\frac{e}{c} \boldsymbol{B}, \\
& \boldsymbol{\Omega}_{i}=\nabla \times\left(G_{2} m_{0 i} \boldsymbol{V}_{i}\right)+\frac{e}{c} \boldsymbol{B} .
\end{aligned}
$$

The equilibrium is therefore governed by the following three coupled equations:

$$
\begin{gathered}
\nabla \times\left(G_{1} m_{0 e} \boldsymbol{V}_{e}\right)-\frac{e}{c} \boldsymbol{B}=a \boldsymbol{V}_{e}, \\
\nabla \times\left(G_{1} m_{0 p} \boldsymbol{V}_{p}\right)+\frac{e}{c} \boldsymbol{B}=a \boldsymbol{V}_{p}, \\
\nabla \times\left(G_{2} m_{0 i} \boldsymbol{V}_{i}\right)+\frac{e}{c} \boldsymbol{B}=b \boldsymbol{V}_{i},
\end{gathered}
$$

where $a$ and $b$ are constants. It is clear from above equations that canonical vorticities of electrons, positrons and ions are proportional to their respective flows.

We take $m_{0 e}=m_{0 p}$, as the electrons and positrons have equal rest masses. The quasi-neutrality condition reads as $e n_{e}=e n_{p}+e n_{i}$ and the current density, using Ampere's Law $\nabla \times \boldsymbol{B}=\frac{4 \pi}{c} \boldsymbol{J}$, is given by

$$
\frac{c}{4 \pi e} \nabla \times \boldsymbol{B}-n_{i} \boldsymbol{V}_{i}=n_{p} \boldsymbol{V}_{p}-n_{e} \boldsymbol{V}_{e} .
$$

Multiplying (8) by $n_{e}$ and (9) by $n_{p}$ and subtracting, we obtain

$$
\begin{aligned}
& G_{1} m_{0 e} \boldsymbol{\nabla} \times\left(n_{p} \boldsymbol{V}_{p}-n_{e} \boldsymbol{V}_{e}\right) \\
& \quad+\frac{e}{c}\left(n_{e}+n_{p}\right) \boldsymbol{B}=a\left(n_{p} \boldsymbol{V}_{p}-n_{e} \boldsymbol{V}_{e}\right) .
\end{aligned}
$$

Using (11), above equation becomes

$$
\begin{aligned}
& \frac{G_{1} m_{0 e} c}{4 \pi e} \nabla \times \nabla \times \boldsymbol{B}-G_{1} m_{0 e} n_{i} \nabla \times \boldsymbol{V}_{i} \\
& \quad+\frac{e}{c}\left(n_{e}+n_{p}\right) \boldsymbol{B}=\frac{a c}{4 \pi e} \nabla \times \boldsymbol{B}-a n_{i} \boldsymbol{V}_{i} .
\end{aligned}
$$

From (10) and (13), the ion flow $\boldsymbol{V}_{i}$ can be written as

$$
\boldsymbol{V}_{i}=\frac{1}{\chi}\left[\nabla \times \nabla \times \boldsymbol{B}-\frac{a}{G_{1} m_{0 e}} \nabla \times \boldsymbol{B}+\Lambda \boldsymbol{B}\right],
$$

where

$$
\chi=\frac{4 \pi e n_{i}}{c}\left(\frac{b}{G_{2} m_{0 i}}-\frac{a}{G_{1} m_{0 e}}\right),
$$

and

$$
\Lambda=\frac{1}{G_{2} \lambda_{i}^{2}}+\frac{1}{G_{1}}\left(\frac{1}{\lambda_{e}^{2}}+\frac{1}{\lambda_{p}^{2}}\right)
$$

$\lambda_{\alpha}=c / \omega_{p \alpha}(\alpha=e, p, i)$ is the skin depth and $\omega_{p \alpha}=$ $\left(\frac{4 \pi e^{2} n_{\alpha}}{m_{o x}}\right)^{1 / 2}$ represents the plasma frequency of $\alpha$ particle. Putting value of $\boldsymbol{V}_{i}$ into (10), we obtain

$$
\nabla \times \nabla \times \nabla \times \boldsymbol{B}-k_{1} \nabla \times \nabla \times \boldsymbol{B}+k_{2} \nabla \times \boldsymbol{B}-k_{3} \boldsymbol{B}=0,
$$


where

$$
\begin{aligned}
& k_{1}=\frac{a}{G_{1} m_{0 e}}+\frac{b}{G_{2} m_{0 i}}, \\
& k_{2}=\Lambda+\frac{a b}{G_{1} G_{2} m_{0 e} m_{0 i}}, \\
& k_{3}=\frac{a}{G_{2}^{2} m_{0 e} \lambda_{i}^{2}}+\frac{b}{G_{1} G_{2} m_{0 i}}\left(\frac{1}{\lambda_{e}^{2}}+\frac{1}{\lambda_{p}^{2}}\right) .
\end{aligned}
$$

This is the triple curl Beltrami equation, a linear sum of three different Beltrami fields, for relativistic $e-p-i$ plasma. If we consider the ions are cold and take the nonrelativistic limit $G_{2}=1$ while the electrons and positrons are hot, the system again self-organizes to triple curl Beltrami field. Let $\boldsymbol{F}_{j}$ (where $j=1,2,3$ ) be the Beltrami field such that

$$
\left\{\begin{array}{cr}
\nabla \times \boldsymbol{F}_{j}=\lambda_{j} \boldsymbol{F}_{j} & (\text { in } \boldsymbol{\Omega}), \\
\mathbf{n} \cdot \boldsymbol{F}_{j}=0 & (\text { on } \partial \boldsymbol{\Omega}) .
\end{array}\right.
$$

Then

$$
\boldsymbol{B}=\sum_{j=1}^{3} C_{j} \boldsymbol{F}_{\boldsymbol{j}},
$$

gives the solution of (15), where $C_{j}^{\prime} s$ are arbitrary constants. The eigenfunctions of the curl operator $\boldsymbol{F}_{j}$ may be represented by Chandrasekhar-Kendall functions (Chandrasekhar and Kendall 1957) in cylindrical geometry and by ABC flow (Yoshida 2010) in slab geometry. The eigenvalues of the curl operator $\left(\lambda_{j}\right)$ may be real or complex (Yoshida and Giga 1990) and these are the solutions of the cubic equation

$$
\lambda^{3}-k_{1} \lambda^{2}+k_{2} \lambda-k_{3}=0 .
$$

The three roots of the above equation are as follows:

$$
\begin{aligned}
& \lambda_{1}=s-\tau+\frac{k_{1}}{3}, \\
& \lambda_{2}=-\frac{1}{2}(s-\tau)-i \frac{\sqrt{3}}{2}(s+\tau)+\frac{k_{1}}{3}, \\
& \lambda_{3}=-\frac{1}{2}(s-\tau)+i \frac{\sqrt{3}}{2}(s+\tau)+\frac{k_{1}}{3},
\end{aligned}
$$

where

$$
\begin{aligned}
& \tau=\left(\frac{q}{2}+\sqrt{D}\right)^{1 / 3}, \\
& s=-\left[\frac{q}{2}-\sqrt{D}\right]^{1 / 3}, \\
& p=k_{2}-\frac{k_{1}^{2}}{3}, \\
& q=\frac{k_{1} k_{2}}{3}-\frac{2 k_{1}^{3}}{27}-k_{3} .
\end{aligned}
$$

When $D=\frac{q^{2}}{4}+\frac{p^{3}}{27}<0$, all the three roots of (21) are real, when $D>0$, then one of the roots $\left(\lambda_{j}\right)$ is real while the others two are complex conjugate pair. When $D=0$, all the roots are real and at least two are equal.
The eigenvalues $\left(\lambda_{j}\right)$ of the curl operator and $k^{\prime} s$ are related by following relations:

$$
\begin{aligned}
& k_{1}=\lambda_{1}+\lambda_{2}+\lambda_{3}, \\
& k_{2}=\lambda_{1} \lambda_{2}+\lambda_{2} \lambda_{3}+\lambda_{1} \lambda_{3}, \\
& k_{3}=\lambda_{1} \lambda_{2} \lambda_{3} .
\end{aligned}
$$

It is evident from (15) that hot $e-p-i$ plasma relaxes to triple curl Beltrami field (TCB) which is a superposition of three Beltrami fields. The coupling of multiple Beltrami fields could create high $\beta$ relaxed equilibrium states. Therefore, multiscale structures can be obtained when hot $e-p-i$ plasma gets the steady state equilibrium. These structures strongly depend on Beltrami parameters $a$ and $b$, the ratios of the canonical momentum of different fluids to their flow, and temperature-dependent mass of the species $G_{1}$ and $G_{2}$. The scale parameters $\lambda_{j}$ characterize the size of structures formed in the system as they are dimensionally equal to the inverse of length. The eigenvalues of the Beltrami functions $\lambda_{j}$ depend on Beltrami parameters and thermal energies $\left(G_{1}\right.$ and $\left.G_{2}\right)$, therefore, the microscopic as well as macroscopic structures can be obtained for different Beltrami parameters and thermal energies of different species. Thus it is possible for $e-p-i$ plasma to relax to three structures, large one corresponding to system size and two small structures of the order of electron skin depth.

To summarize, we have investigated the steady state solution of relativistic $(e-p-i)$ plasma. It is found that for either the case, when all the plasma constituents are relativistically hot or when the electrons and positrons are hot and ions are cold, the relaxed equilibrium can be expressed by triple curl Beltrami field. The triple curl Beltrami field admits the formation of three relaxed structures of different scale sizes. These structures are strongly controlled by thermal energies of the plasma particles and Beltrami parameters. Development of such structures in relativistically hot $e-p-i$ plasmas could be used to understand the formation of relativistic jets on large scales in different radio galaxies, quasars or radio pulsars. Our results may also be applied to study the structure formation in laboratory plasmas when ultrarelativistic laser beams are used to produce relativistically hot plasmas. The detailed analysis of the effect of thermal energies of the plasma particles on the formation of relaxed structures, on other physical parameters of interest like magnetic fields, flows, helicities, field and flow energies need to be addressed and will be dealt with elsewhere.

\section{References}

Berezhiani, V. I., El-Ashry, M. Y. and Mofiz, U. A. 1994 Theory of strong-electromagnetic-wave propagation in an electron-positron-ion plasma. Phys. Rev. E 50, 448452 . 
Berezhiani, V. I., Garuchava, D. P. and Shukla, P. K. 2007 Production of electron-positron pairs by intense laser pulses in an overdense plasma. Phys. Lett. A 360, 624628.

Berezhiani, V. I. and Mahajan, S. M. 1994 Large amplitude localized structures in relativistic electron-positron ion plasma. Phys. Rev. Lett. 73, 1110-1113.

Berezhiani, V. I. and Mahajan, S. M. 1995 Large relativistic density pulses in electron-positron-ion plasmas. Phys. Rev. E 52, 1968-1979.

Berezhiani, V. I., Mahajan, S. M., Yoshida, Z. and Ohhashi, M. 2002 Self-trapping of strong electromagnetic beams in relativistic plasmas. Phys. Rev. E 65, 047402-047405.

Berezhiani, V. I., Tsintsadze, L. N. and Shukla, P. K. 1992a Influence of electron-positron pairs on the wakefields in plasmas. Phys. Scr. 46, 55-56.

Berezhiani, V. I., Tskhakaya, D. D. and Shukla, P. K. 1992b Pair production in a strong wake field driven by an intense short laser pulse. Phys. Rev. A 46, 6608-6612.

Bhattacharyya, R., Janaki, M. S. and Dasgupta, B. 2003 Relaxation in electron-positron plasma: a possibility. Phys. Lett. A 315, 120-125.

Björnsson, G. and Svensson, R. 1992 Hot pair-dominated accretion disks. Astrophys. J. 394, 500-514.

Chandrasekhar, S. and Kendall, P. C. 1957 On force-free magnetic fields. Astrophys. J. 126, 457-460.

Chen, K. and Ruderman, M. 1993 Pulsar death lines and death valley. Astrophys. J. 402, 264-270.

Helander, P. and Ward, D. J. 2003 Positron creation and annihilation in tokamak plasmas with runaway electrons. Phys. Rev. Lett. 90, 135004-135007.

Iqbal, M., Berezhiani, V. I. and Yoshida, Z. 2008 Multiscale structures in relativistic pair plasmas. Phys. Plasmas 15, 032905-032910.

Iqbal, M. and Shukla, P. K. 2011 Relaxation of a magnetized electron-positron-ion plasma with flow. Phys. Lett. A 375, 2725-2727.

Lakhina, G. S. and Buti, B. 1981 Generation of a d.c. field by nonlinear electromagnetic waves in relativistic plasmas. Astrophys. Space Sci. 79, 25-36.

Liang, E. P., Wilks, S. C. and Tabak, M. 1998 Pair production by ultraintense lasers. Phys. Rev. Lett. 81, 4887-4890.

Lightman, A. P. and Zdziarski, A. A. 1987 Pair production and compton scattering in compact sources and comparison to observations of active galactic nuclei. Astrophys. J. 319, 643-661.

Lominadze, D. C., Machabeli, G. Z., Melikidze, G. I. and Pataraya, A. D. 1986 Magnetospheric plasma of a pulsar. Sov. J. Plasma Phys. 12, 712-721.

Machabeli, G. Z., Luo, Q., Melrose, D. B. and Vladimirov, S. 2000a A new mechanism for pulsar gamma-ray emission. Mon. Not. R. Astron. Soc. 312, 51-56.

Machabeli, G. Z., Luo, Q., Vladimirov, S. V. and Melrose, D. B. 2002 Quasilinear diffusion as a result of modulational instability in the pulsar plasma. Phys. Rev. E. 65, 036408.

Machabeli, G. Z., Vladimirov, S. V. and Melrose, D. B. 1999 Nonlinear dynamics of an ordinary electromagnetic mode in a pair plasma. Phys. Rev. E. 59, 4552-4558.

Machabeli, G. Z., Vladimirov, S. V., Melrose, D. B. and Luo, Q. 2000b Particle acceleration by a fast ordinary mode in an electron-positron plasma. Phys. Plasmas 7, 1280 1286.

Mahajan, S. M. and Yoshida, Z. 1998 Double curl Beltrami flow: diamagnetic structures. Phys. Rev. Lett. 81, 48634866.
Mahmood, S. and Ur-Rehman, H. 2009 Electrostatic solitons in unmagnetized hot electron-positron-ion plasmas. Phys. Lett. A 373, 2255-2259.

Marklund, M. and Shukla, P. K. 2006 Nonlinear collective effects in photon-photon and photon-plasma interaction. Rev. Mod. Phys. 78, 591-640.

Misner, C. W., Thorne, K. S. and Wheeler, J. A. 1980 Gravitation. San Francisco: Freeman.

Popel, S. I., Vladimirov, S. V. and Shukla, P. K. 1995 Ionacoustic solitons in electron-positron-ion plasmas. Phys. Plasmas 2, 716-719.

Rees, M. J. 1983 The Very Early Universe (ed. W. G. Gibbson, S. W. Hawking and S. Siklos) Cambridge: Cambridge University Press.

Reynolds, C. S., Fabian, A. C., Celotti, A. and Rees, M. J. 1996 The matter content of the jet in M87 - evidence for an electron-positron jet. Mon. Not. R. Astron. Soc. 283, 873-880.

Rizzato, F. B. 1989 Weak nonlinear electromagnetic waves and low-frequency magnetic-field generation in electronpositron-ion plasmas. J. Plasma Phys. 40, 289-298.

Shah, A. and Saeed R. 2009 Ion acoustic shock waves in a relativistic electron-positron-ion plasma. Phys. Lett. A 373, 4164-4168.

Shatashvili, N. L., Javakhishvili, J. I. and Kaya, H. 1997 Nonlinear wave dynamics in two temperature electronpositron-ion plasma. Astrophys. Space Sci. 250, 109-115.

Shukla, P. K. 2005 Beltrami fields in a three-species magnetoplasma. Phys. Lett. A 334, 205-207.

Shukla, P. K. and Mahajan, S. M. 2004a Relaxed states in magnetized pair plasmas. Phys. Scr. T113, 151-152.

Shukla, P. K. and Mahajan, S. M. 2004b Formation of large scale structures in dusty magnetoplasmas. Phys. Lett. A 328, 185-188.

Surko, C. M., Leventhal, M., Crane, W. S., Passner, A., Wysocki, F., Murphy, T. J., Strachan, J. and Rowan, W. L. 1986 Use of positrons to study transport in tokamak plasmas. Rev. Sci. Instrum. 57, 1862-1867.

Surko, C. M. and Murphy, T. 1990 Use of the positron as a plasma particle. Phys. Fluids B 2, 1372-1375.

Svensson, R. 1994 The nonthermal pair model for the Xray and gamma-ray spectra from active galactic nuclei. Astrophys. J. Suppl. Ser. 92, 585-592.

Takahara, F. and Kusunose, M. 1985 Electron-positron pair production in a hot accretion plasma around a massive black hole. Prog. Theor. Phys. 73, 1390-1400.

Taylor, J. B. 1974 Relaxation of toroidal plasma and generation of reverse magnetic fields. Phys. Rev. Lett. 33, 1139-1141.

Taylor, J. B. 1986 Relaxation and magnetic reconnection in plasmas. Rev. Mod. Phys. 58, 741-763.

Tinkle, M. D., Greaves, R. G., Surko, C. M., Spencer, R. L. and Mason, G. W. 1994 Low-order modes as diagnostics of spheroidal non-neutral plasmas. Phys. Rev. Lett. 72, $352-355$.

Yoshida, Z. 2010 Nonlinear Science. Dordrecht: Springer.

Yoshida, Z. and Giga, Y. 1990 Remarks on spectra of operator rot. Math. Z. 204, 235-245.

Yoshida, Z. and Mahajan, S. M. 1999 Simultaneous Beltrami conditions in coupled vortex dynamics. J. Math. Phys. 40, 5080-5091.

Yoshida, Z., Mahajan, S. M., Ohsaki, S., Iqbal, M. and Shatashvili, N. 2001 Beltrami fields in plasmas: highconfinement boundary layers and high beta equilibria. Phys. Plasmas 8, 2125-2131. 